\title{
Correlation between Self-Reported Depression and Adolescent Obesity
}

\author{
Anna Tharakan ${ }^{1}$, Michael Horn'1, and Justin McCrakin¹ \\ ${ }^{1}$ Jenks High School, Jenks, OK, USA
}

\section{$\underline{\text { ABSTRACT }}$}

This research study focused on the correlational relationship between self-reported depression levels and BMI. Health risks and suicidal levels have increased on a global scale due to slowly rising levels of obesity and depression. Past research has been conducted at a global level, only slightly touching on the direct correlation, finding that if one shows an increase, the other will as well1. However, there has been a lack of research focused on the local scale, specifically in suburban high schools in the Midwest. The author gave participants a personally constructed survey with the choice of an interview, testing their perception between the two variables. BMI along with results from a professionally developed depression test were reported, allowing for statistical analysis to be run between the two. This direct correlation, along with other potential factors receiving $\mathrm{r}$ values ranging from 0.04 to 0.5 , found a few statistically significant relationships. This research led to surprising conclusions, with evidence for necessary further research.

\section{Introduction}

Throughout history, the way one appears has always bore importance to societal values and their overall quality of life. Societal pressures have continuously increased, with stress on body image and self health. More have attempted to conceal their mental appearance, which has caused an onset of depression. Depression can be the result of psychological and physiological events in one's lifetime, defined as "the result of habitual feelings of pessimism and hopelessness ${ }^{2}$." Any number of long term events, such as health disorders or upbringing, can alter mental health and increase depression related incidents.

The rates of depression have increased by significant levels in the United States, especially among adolescents. According to the National Survey on Drug Use and Health3, in 2005 and 2015, depression levels were 6.6 and $7.3 \%$, respectively. This increase was specifically evident in children ages 12 to 17 and individuals from vulnerable socioeconomic backgrounds. According to the World Health Organization4, "mental illness caused by depression will be second only to HIV in the burden it places on the world by the end of this [next] decade." Depression is more commonly viewed as a terminal disease; however, not enough is being done to resolve the problems that arise from these rapidly increasing numbers. The earlier onset of depression has been associated with detrimental consequences, as the study conducted by Peter M. Lewinsohn ${ }^{5}$, author at the Oregon Research Institute, showed that those who suffered depression at an earlier age had longer episodes of depression (ranging over eight weeks) and a higher chance of relapsing to a depression state -- $12 \%$ in one year.

This increase in depression has not come without consequences. Untreated depression has become a very strong risk factor for suicidal behavior, and recent studies show that "suicide attempts have increased in recent years, especially among young women6." Depression induced suicide has become the third leading cause of teenage death7. According to the Youth Risk Behavior Surveillance Survey8, over 20\% of youth had attempted suicide in 2018. These skyrocketing numbers are not only higher than ever before, but solutions have not been adequately addressed. The high risks that come with increased depression are now soaring and ignoring these growing conditions can be a mistake. 
Another public health problem that seems to follow the same trend as depression is obesity. Obesity levels are often measured with the Body Mass Index, or BMI, which divides an individual's weight by their height in association to age. Individuals are then classified into four categories: underweight, normal, overweight, obese ${ }^{2}$. An increase in BMI has begun to affect younger populations, and it was estimated that in $201015.2 \%$ of 18 year-olds have suffered or were currently suffering from an increase in BMI and even more were considered to be overweight. The prevalence of adolescent obesity has increased from 1994 to 2014, almost doubling in average BMI ${ }^{9}$. According to the National Health and Nutrition Examination Survey ${ }^{9}$ in 2014, 70.2\% of the national population was considered overweight or obese. This immense change in BMI over time has affected some of the most vulnerable groups in the United States, as the International Journal of Obesity found "participants in the lowest socioeconomic status (SES) neighborhoods were more likely to experience increases in BMI than participants in the highest SES neighborhoods ${ }^{21}$." Since these two important trends have shown a significant increase within the same timespan, a logical conclusion could be made between the potential correlation and effects between the two. However, the research that has been conducted on this correlation shows conflicting results. The distinct relationship between depression and weight class has not been entirely researched and the relevant literature did not provide a specific conclusion. One study conducted in 2015 by Child Psychiatry and Human Development ${ }^{1}$ used the early stage comparison between the percentage of obese or overweight people and their depression rates. When testing the correlation, the study found a direct relation between the variables.

The correlations were tested with diverse populations, however limited on the breadth of factorial results. Brandon Respress, doctor and author at the University of Texas, found that certain races, specifically black and hispanic, were at a higher risk of depression and BMI at younger ages in a school environment ${ }^{24}$. Limited research has been conducted on how BMI and depression interchangeably affect these factors. The only factors that have been commonly discussed have been socioeconomic levels (SES), as the American Journal of Community Psychology tested the link, finding that a lack of social integration, common in lower SES adolescents, led to higher depression levels ${ }^{25}$.

The few studies conducted on a high school scale found high corresponding values. In a 2016 research study testing the relationship between BMI and depression among Iranian high school female students saw a 55\% correla$\operatorname{tion}^{26}$. However, this limited scope failed to take into account other races or genders, leading to compromised results.

What remains unclear about the link between BMI and depression rates is the strength of the correlation between the two. Studies have focused on the potential effects of depression or have simply compared the rising numbers; however, minimal research has been conducted to test these effects on a local scale, such as in high schools. A lack of a consistent scope fails to attribute to the results of the correlative studies, so creating an environment that tests various factors will create a new set of results desperately needed. Previous studies failed to look at the firstperson perspective of high schoolers, therefore, this study attempts to provide insight into this gap. A lack of research has been done to examine specific factors and their overwhelming effects, failing to connect BMI and depression and analyze the results. The extent to which each factor influences the other is of importance, and it begs the question: to what extent, if any, does the correlational effect between self-reported depression and BMI levels play a factor at a suburban high school? It is hypothesized that a large correlation between depression levels and BMI will be found, showing that whenever the depression rates increase or are high, the BMI levels will follow. Due to the limited nature of the study, the research is limited to a current correlation, rather than effects over a longer period of time. It would be beneficial to society overall to conduct a correlational study in which both qualitative and quantitative data were to be collected, giving a better picture of the effect of the rising adolescent depression on the world. The larger picture, provided through the research, will serve to prove that this correlation can explain rising levels of BMI and depression in adolescents in the status quo. 


\section{Method}

A correlational study was selected as the method as it searches for the relationship between two variables that are not controlled by the researcher. Instead of having an independent and dependent variable, a correlational study tests the link between two existing ideologies. This method will also help alleviate the problem of experiments affecting how a participant, knowing they are under a stressful situation, may react ${ }^{10}$.

This research study, which attempted to identify a link between depression levels and BMI levels, consisted of two components: a school-wide randomized survey and selective interviews. This specific style of research survey was selected as it was viewed as the quickest and most efficient way of gathering data. According to the American Psychological Association ${ }^{11}$, surveys provide the researcher with an opportunity to gather answers to questions from a large population in a quick and noninvasive manner. Considering that the study was held at a high school over a short timespan, reaching a large audience was an essential factor.

The survey was open to the entire student population of a midwestern suburban high school. SurveyMonkey, a popular survey developer, was utilized for the easiest breadth of results. In order to reach as many students as attainable, not only was the survey link spread by word of mouth, but additionally 40 classes ranging from 10th-12th grade were randomly selected from a comprehensive list. While not all teachers complied, efforts were taken to ensure complete reach, and almost 35 classes responded to the efforts. The survey was held open for the duration of one month for optimal but timely results.

The survey contained two main question types, the first being basic demographic questions and the second being directly related to the survey (see Appendix A for questions). The initial demographic information, such as age and gender, was collected along with socioeconomic status (SES) factors, such as family income and living area. The section containing information related to the survey included the participant filling out an affiliated depression test. This test is less than 18 questions and has been accredited by several institutions, one being the University of Florida. The test was created by John M. Grohol12, PhD, the founder of PsychCentral, and provides a complete questionnaire that can directly determine the depression status of the participant. This depression test analyzes various issues, such as time management and depression tendencies. With the large variety of tests available on the internet, this specific depression test not only seemed the most concise but also one of the most accredited. At the end of the test, the participant is given a number that correlates to a scale of normal, sad, and depressed (see Appendix B for scale). The survey asked for the numerical value of the participant's result, rather than the position given, for a more accurate determination of the strength of the correlation.

The research-related section continued with questions such as rating their own depression and choosing some factors that they believe would lead to this. The survey continued by asking for the height and weight of the participant. This information aided in calculating BMI for each specific individual and allowed for a test of the possible link between depression rate and BMI. Then, for more accuracy, BMI was calculated using an online BMI calculator ${ }^{13}$. All results of the survey were kept anonymous for the privacy of the participant; however, for those who were willing to be interviewed, anonymity was eliminated. In order to sign up for the interview process, they provided their email address so the researcher had access to their contact information if further information was needed.

The secondary component of the research was selective interviews. Once the results of the surveys were analyzed and put together, those that allowed for permission were contacted for a face-to-face interview. The major purpose of the interview was to uncover further details about their personal results and different factors that they might find to be important in relation to their depression level or BMI level. Many of the questions asked what relation the environment of high school has on that individual or how their answers impacted them in a broader aspect, focusing on the qualitative responses (see Appendix A for general interview questions). Looking at the degree to which mental health played in people's lives or the extent to which their BMI may have had a negative effect is helpful when determining a connection. An interview also helps explain whether large values on the depression test are from a certain event or an overall lifetime. The outline of the research project did not allow testing this correlation over a longer duration, so the data is restricted to the current status of depression and physical attributes of that participant. 
The test to determine the depression level would also be self-reported as it did not fit into the span of the research to allow each participant to be medically tested.

Throughout the data collection, the potential of a suicidal participant being found was relatively high, but because of the nature of this anonymous survey, there was not an opportunity to identify the participant. A suicide helpline was located at the bottom of the survey in order to help those participants that may be struggling. The survey was initiated with an online consent form that confirms that the participant is signing up for the use of their confidential information in the research (see Appendix A). If a participant continued on with the survey, this was counted as admission towards the consent form. Those that were interviewed were given a consent form that they had to sign in order to continue the process (see Appendix A).

\section{Results}

The following graphic representations were created from analytics by Survey Monkey and data charts using Microsoft Excel. The correlation coefficient ( $\mathrm{r}$ value) was calculated for each data set and further indicated through statistical analysis. Out of the 160 participant responses, six had to be eliminated from the study for various reasons. A few failed to provide some of the essential information, such as the weight needed to calculate BMI or incomplete depression test. Other results were proven to be false, as numerical values of the depression test were entered that could not be achieved.

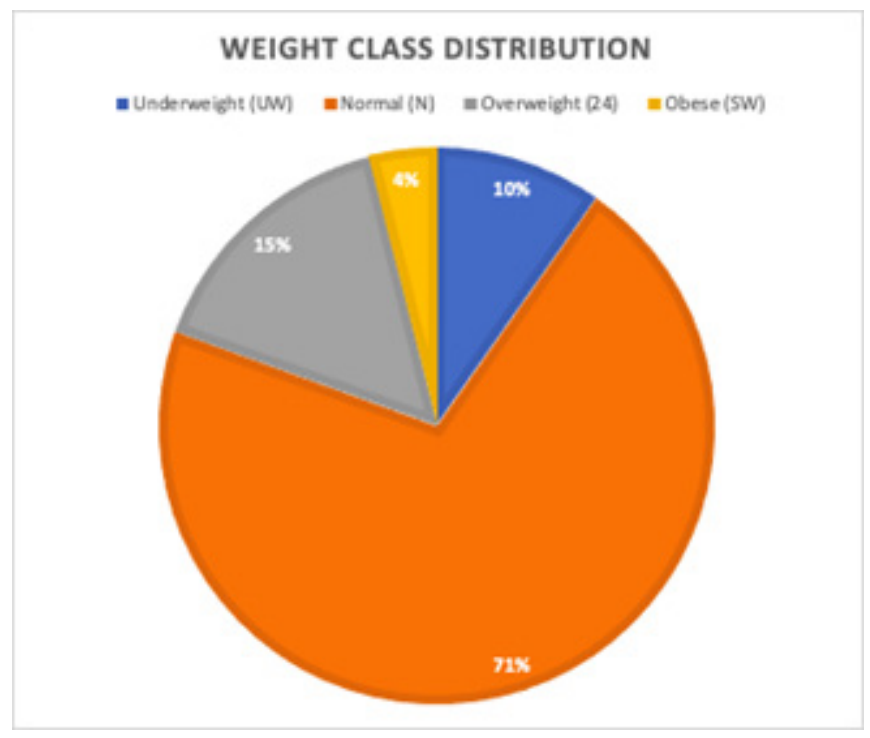

Figure 1.1. Weight Class Distribution. 


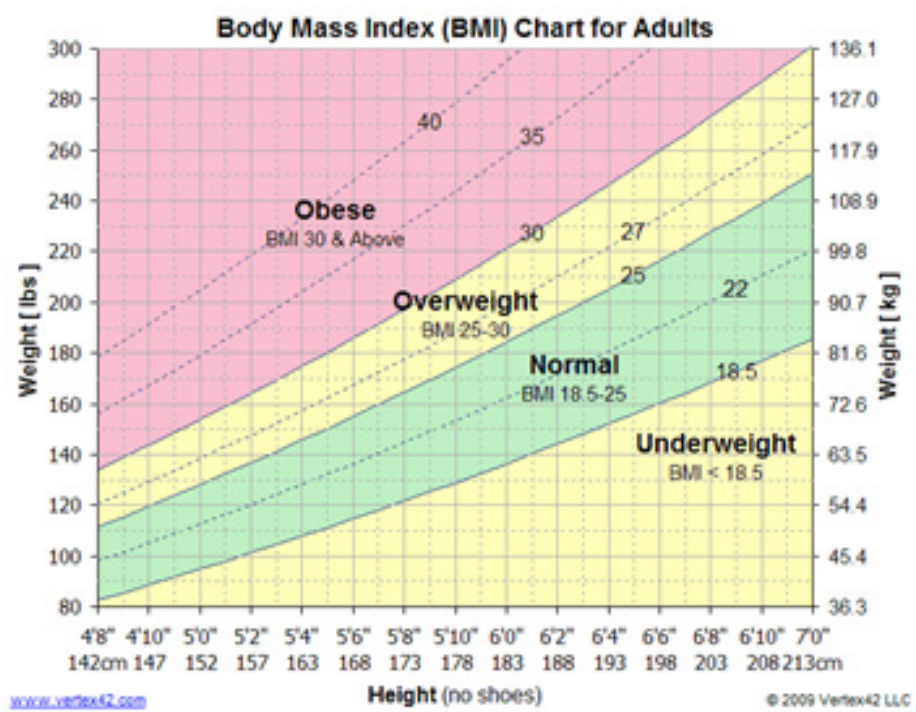

Figure 1.2: Body Mass Index (BMI) for Adults.

All recorded BMIs were organized into 4 weight classes, using the scale provided by the CDC (FIGURE 1.2). The weight classes were found to be underweight (UW), normal(N), overweight (OW), and severely overweight/obese (SW). They were then used to compare the different data sets and test for a potential correlation. Figure 1.1 exhibits the weight class distribution of the participant field, with a large majority (71\%) shown having a normal weight distribution. This fits in with a BMI from 18.5 to 25 .

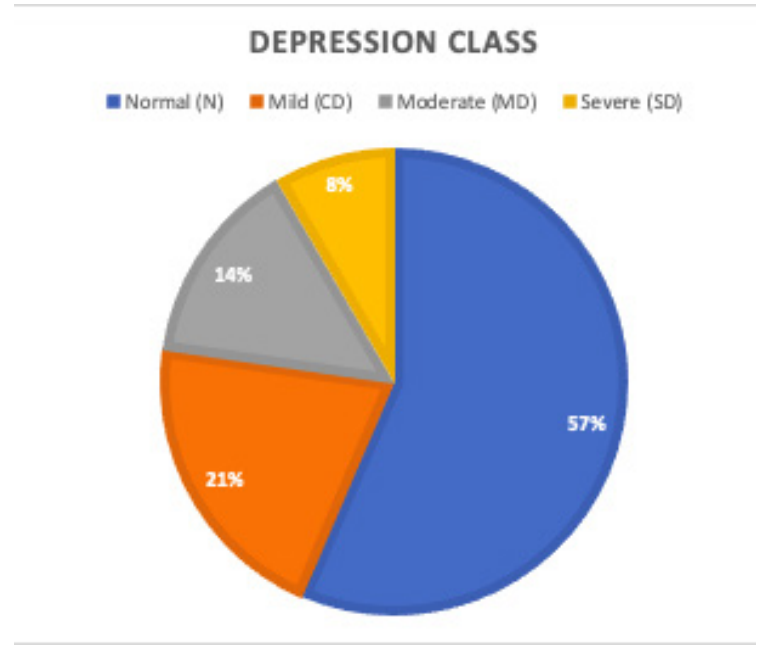

Figure 1.3. Depression Class.

Distribution from the different depression levels fit in similar with BMI. 57\% were found to have normal depression levels (0-18). A large percentage (21\%) had a mild level (18-35). This seemed expected from a normal population as seen in the data. 


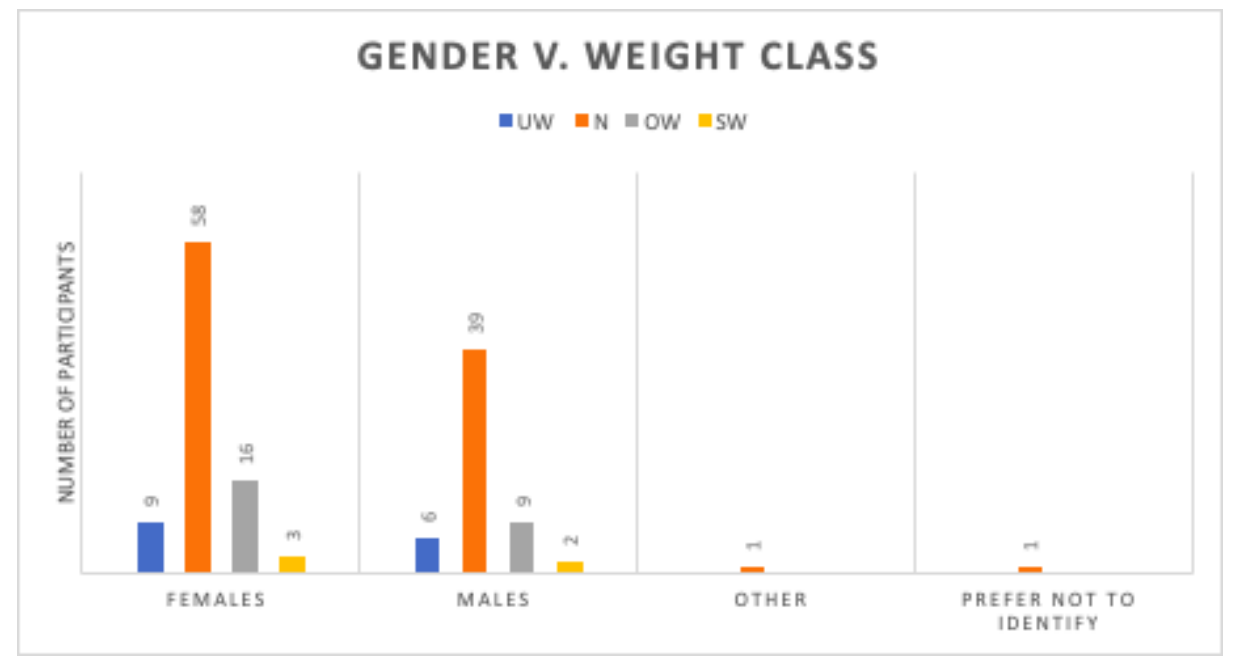

Figure 2.1. Gender vs. Weight Class.

This graphic representation compares different gender identifications with their respective weight classes. The weight classes were determined from the BMI, ensuring accurate results. The correlation between the two are limited (1.8\%), but the display of limited data shows reason for analysis. Sixty percent of the participant population identified as female, and a majority were found to be of a normal weight class, finding an overall conclusion that gender has no effect on weight class. A similar correlation was viewed with gender and depression levels $(-4.3 \%)$, finding that males are more commonly found to have higher depression levels than women

\begin{tabular}{|l|l|l|}
\hline 1. UW N & $3 . \mathrm{NN}$ & 5. OW N \\
\hline 10 & 84 & 22 \\
\hline 2. UW MD & $4 . \mathrm{N} \mathrm{MD}$ & 6. OW MD \\
\hline 5 & 24 & 8 \\
\hline
\end{tabular}

Figure 3.1

BMI and depression were combined in order to find a more comparative stance with other potential factors. The different weight class categories consisted of underweight (UW), normal weight (N), and overweight or obese (OW). They were combined with depression classes of normal to mild depression (0-21) and moderate to severe (MD). The participants were fit into 6 different categories depending on their results, and the numbers above show the number of participants in each category. $55 \%$ of the participants were seen to be in the middle of the scale, having both a normal weight and normal depression level. However, more outstanding numbers were seen with those that fit into category $5(14 \%)$. 


\section{HISPANIC}

$$
-1=2=3=4
$$

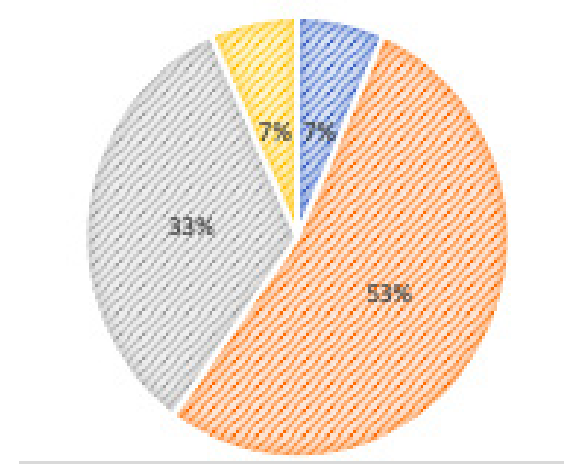

Figure 3.2

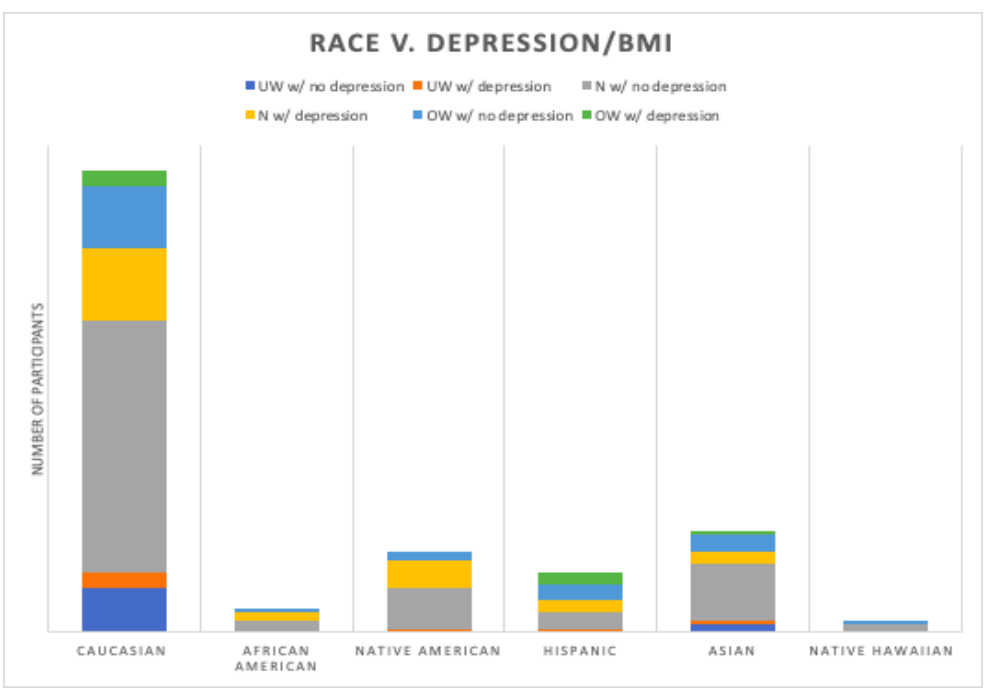

Figure 3.3

Figure 3.3 portrays the correlation between race and a combination of depression and weight class. The graph above is similar to the comparison of weight class, yet shows the consistent correlation when compared to depression as well $(\mathrm{r}=0.104)$. Higher values are seen between the median and the average, with section percentages higher when compared with solely weight class. One interesting aspect of the data was the percentage of specific races to the data labels. For example, $40 \%$ of those that identified as Hispanic were overweight, while only $18 \%$ of the caucasian class had the same results (see Appendix $\mathrm{C}$ for race and depression graphs). A conclusion can be drawn that, from the collected data, Hispanics have a higher BMI than other ethnicities, closely followed by Native Hawaiian. When looking toward the example of Native Americans, the split between the normal weight with and without depression was $50 \%$ and $35 \%$, respectively. 


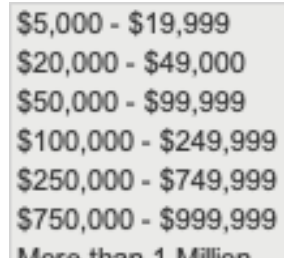

\begin{tabular}{|r|r|}
\hline $4.38 \%$ & 7 \\
\hline $9.38 \%$ & 15 \\
\hline $26.25 \%$ & 42 \\
\hline $38.13 \%$ & 61 \\
\hline $17.50 \%$ & 28 \\
\hline $1.88 \%$ & 3 \\
\hline $2.50 \%$ & 4 \\
\hline
\end{tabular}

Figure 4.1. Annual Income Distribution

Another unique aspect from the layout of the parental annual income was the percentage that was in each category. An overwhelming majority was seen to have an average annual income of $\$ 100,000$ to $\$ 749,999$, with the median being $\$ 100,000$ to $\$ 249,999$. This is significantly higher than the annual income of the average household in the United States, which is $\$ 61,937^{14}$. When searching the median income for the suburban high school, Census Reporter found the median to be $\$ 76,625$ as of $2018^{15}$. The median is significantly higher than the averages for the nation, creating belief of bias or limited data.

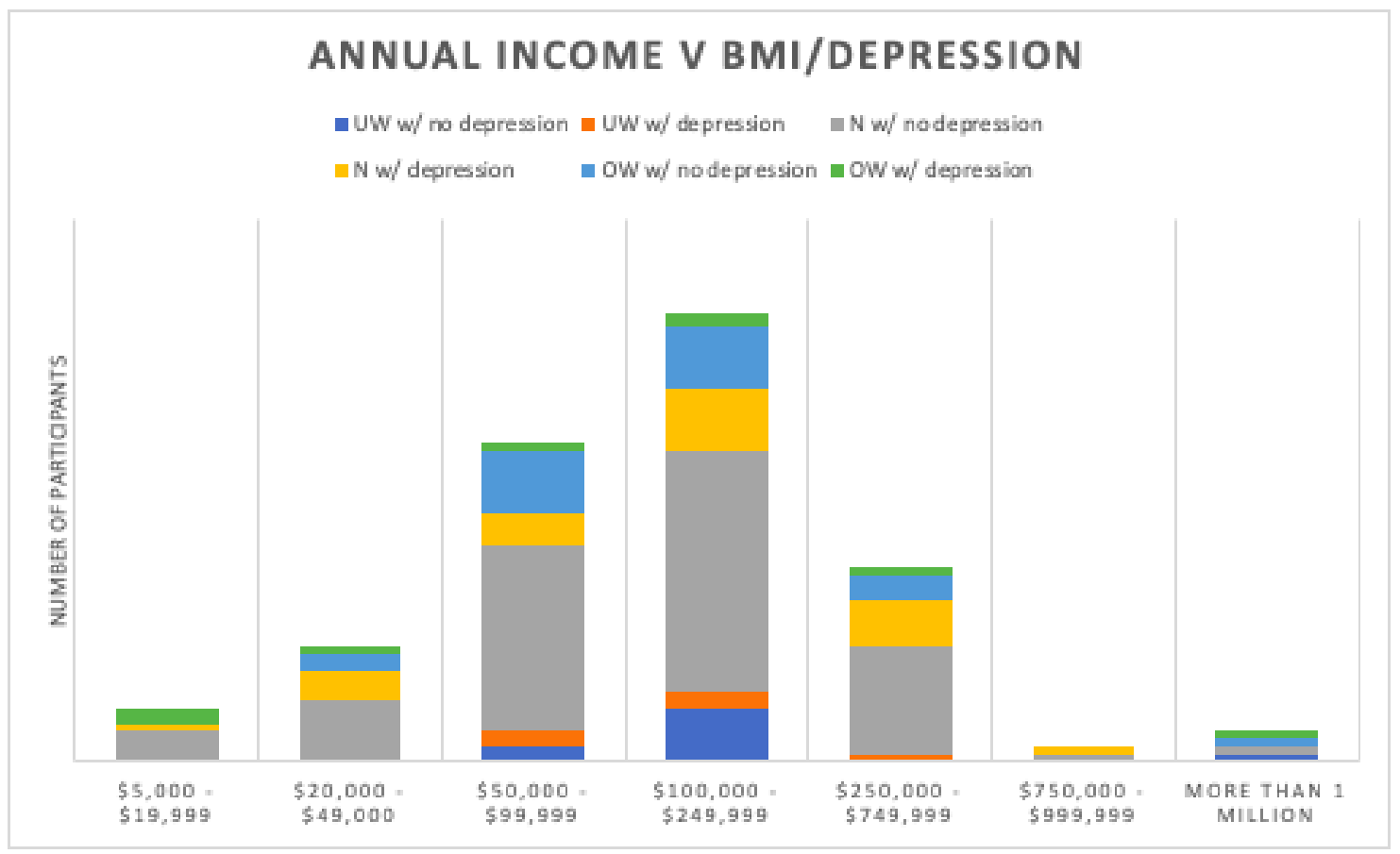

Figure 4.2. Annual Income vs. BMI/Depression.

The correlation between annual income and BMI/depression, when combined, was higher than other factors in this study (-6.2\%). While still not a large percentage, there seems to be more participants that fit outside of the norm of a normal weight class with no depression. This aligned with data from the International Journal of Obesity, as they found that $57.6 \%$ those in a lower socioeconomic status (SES) $(>\$ 49,999)$ had a larger BMI, the largest percentage out of the SES groups ${ }^{21}$. The limitation in the data creates a lower belief of the correlation. When looking at the national average category ( $\$ 50,000$ - $\$ 99,999), 24 \%$ of the data population was found to be overweight compared to the $64 \%$ normal weight (See Appendix C for Annual Income v BMI). 


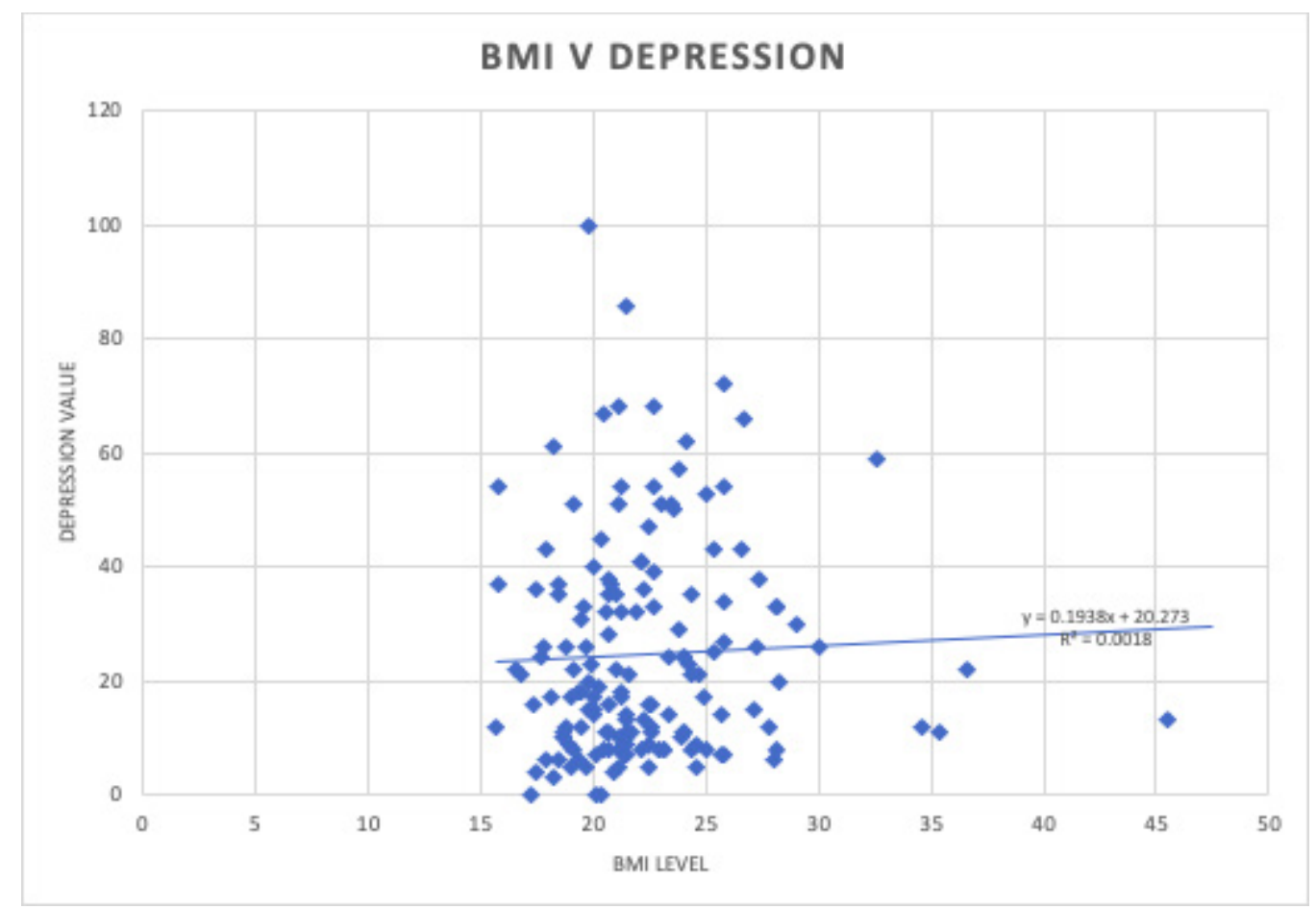

\section{Figure 5.1}

When comparing the graph of BMI levels to depression levels, the correlation seems to be very low. The calculated $\mathrm{r}$-value was 0.042 , showing a low percentage of those that have a higher BMI to have a high depression level. The tested population had limited responses, leading to a small breadth of results. When comparing weight and depression classes, a slightly higher correlation (14.4\%) was seen, but still not significant to draw a conclusive result.

\section{SELF-REPORTED V REAL WEIGHT CLASS}

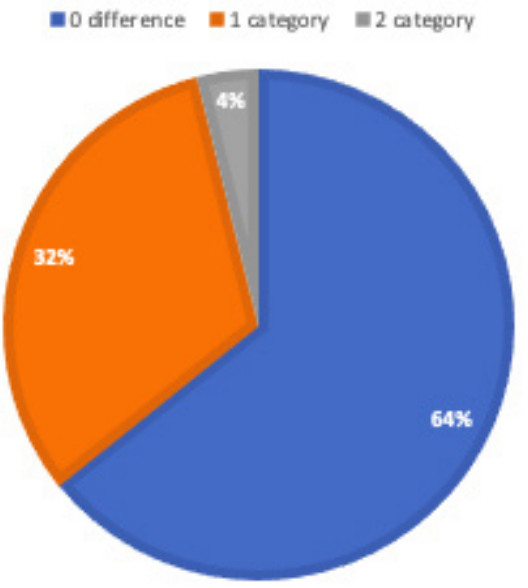

\section{Figure 6.1}

After comparing the self-reported weight classes to those calculated from BMI, there was only a $23.3 \%$ correlation. $32 \%$ misdiagnosed by one class, versus $4 \%$ by two or more. $56 \%$ estimated a weight class lower than the calculated class versus $44 \%$ that overestimated. 


\section{Would you consider yourself depressed, anxious, or of no problem?}

Answered: 160 Skipped: 0

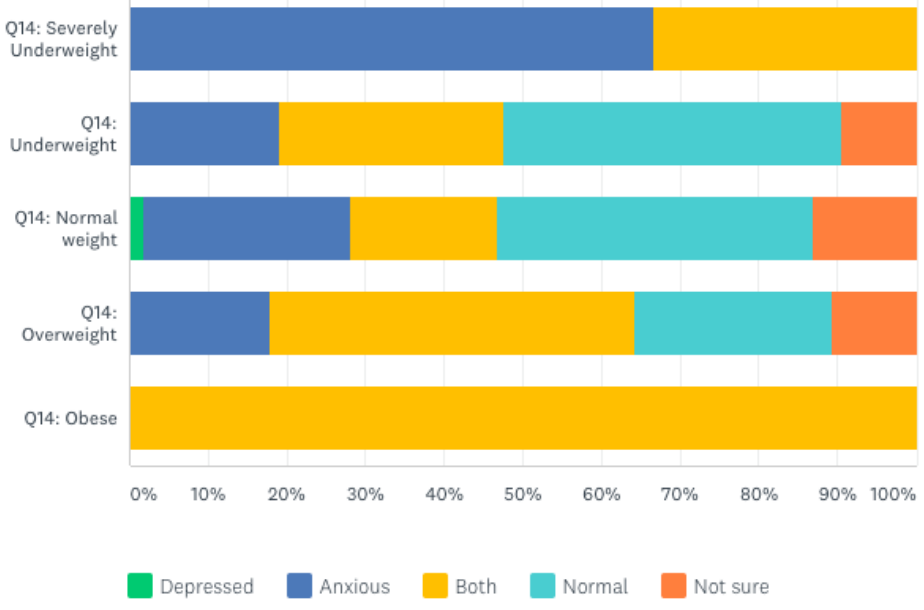

\section{Figure 7.1}

Participants were asked what weight class they thought they were under and their mental status. When looking at the graph, many of those that were overweight or obese also had a higher self-reported depression value. All of those that reported to be obese also stated that they felt both depressed and anxious, later confirmed on the scale provided to the depth of depression effect. The average for those considered obese was 73 (on a scale of 0 to 100), a considerably high value. This high percentage shows that while there was a small number of those that reported to be obese (17), all felt depressed on some level.

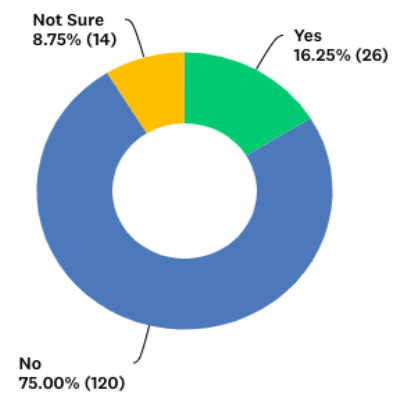

\section{Figure 7.2}

When asked if clinically diagnosed with depression, a higher than expected value of $16.25 \%$ responded yes. 
Have you ever wished serious harm on yourself or others?

Answered: 159 Skipped: 1

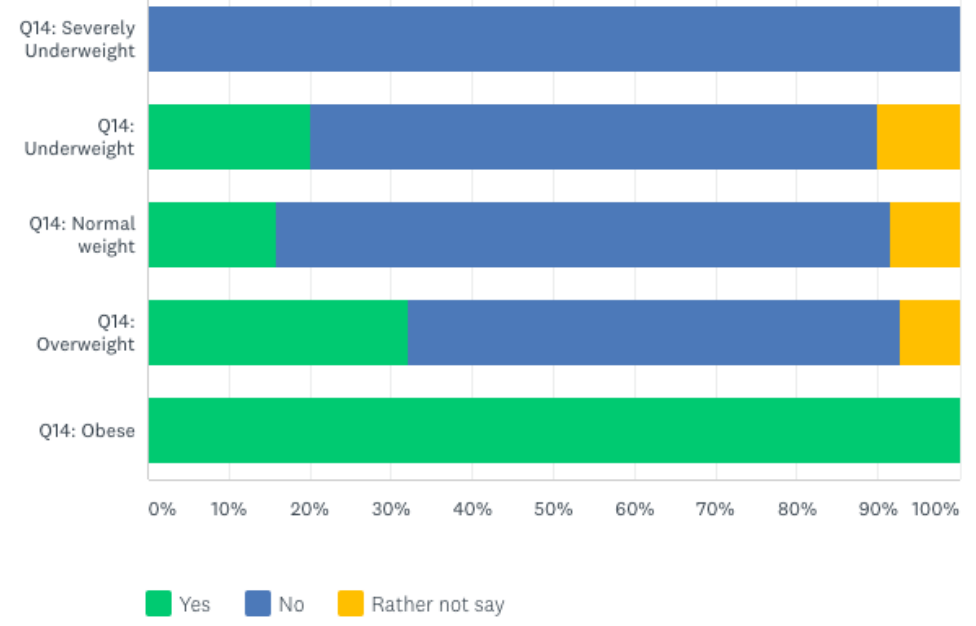

\section{Figure 7.3}

$19.5 \%$ reported that they had wished serious harm on themselves (Appendix C). Furthermore, all seven of those that reported obese stated that they had in fact attempted sucide. Furthermore, $34 \%$ of those considered overweight were found to have answered yes. This percentage steadily decreases, as only $20 \%$ of all of those considered "normal" had selected yes. This decreasing number is attributed to the growing percentage of those with an increased BMI and an increased self-reported depression.

\section{Discussion}

The analysis generates salient discoveries; however, fails to affirm the hypothesis. Due to the lack of correlation found, the data does not show a large distribution in the outcomes. Many of the participants were similar in nature, leading to a limited field. The analysis establishes that although there are multiple factors that can play a role in determining depression and weight levels of an individual, no one factor is solely responsible for the relationship between BMI and depression.

Although the hypothesis was disproved, interesting relationships were discovered. For example, in comparison between race and weight class, $40 \%$ of Hispanics were seen to be overweight, a rather large percentage. This aligned with data provided by the US Department Office of Minority Health, in which found that Hispanics were "30\% more likely to be overweight than their non-Hispanic counterparts" ${ }^{16}$. More research can be conducted to analyze the specific correlation of specific races between BMI or depression, as potentially looking at a larger participant field can broaden potential results of a witnessed correlation.

Gender was seen to play no role in the correlation, as an equal distribution between males and females were observed. However, the annual income distribution alluded to the limited scope of the study. The average income was twice as high as the national average, with a low correlation in the data. Many conflicting studies allude to which socioeconomic status (SES) has the highest BMI, so further exploring potential levels of income and its effect on BMI may show which SES are most at risk. Furthermore, the correlation between perceived and genuine weight class was low, with over $73 \%$ of the participants underestimating or overestimating self-health.

The average in both BMI and depression were normal, with average BMI being 22.3. This is on the lower end of the scale, as a normal BMI range is between 18.5 and 24.9. The average depression level, ranging from values 
of 0-86, was 24.6. This is considered to be mild depression on the scale, as the range is between $22-35.18 .75 \%$ of participant population were found to be overweight or obese (calculated from BMI). This aligned with data found by the National Health and Nutrition Examination Survey, as the percentage was $15.6^{9}$. It was also found $24.02 \%$ of participants were depressed from the data, ranging from a score of 18 and above in the given depression test.

The analysis does not answer the question: to what extent does the correlational effect between self-reported depression and BMI levels play a factor at a suburban high school? The correlational effect is limited (4.2\%). The selected participants had relatively similar backgrounds and therefore did not allow for the question to truly be answered in full. Assumptions can be made about those that fit into the small category of results presented: caucasians with an annual income over $\$ 100,000$, with relatively normal BMIs. Due to the relatively large percentages of data for this participant type, a conclusion can be drawn from this group, but not the larger percentage of those seen at the suburban high school, much less society as a whole.

\section{Implications}

When looking towards the practical indications of the study, the lack of a correlation can help look towards further research studies to solve some of the largest implications of the problem. With the clear societal pressure on individuals that is seen through how people perceive their depression status, more can be done on a local or school level to fix this perception. Currently, many are overlooking these rising levels, but if the correlation between certain minorities or social classes are further found, changes can be made to implement a program to change common perceptions. School was found to be one of the largest points of mental and physical stress, therefore alleviating potential pressures may help solve crucial aspects of the problem.

The common stigma between how many perceive their health and how it appears persisted throughout the study, with alarmingly high levels of potential suicides (19.5\%) and incorrect estimations of their health. Programs to fix the health stigma ought to be implemented to ensure the livelihood of the younger population. Furthermore, participants had a false image of self-health, advocating for larger stress on the role of societal perception on adolescents.

This study proves that certain factors are more responsible than others in this correlation. Due to increased levels for race and SES levels, this finding shows that those in certain conditions are more susceptible to higher depression and BMI levels than others. Higher attention needs to be paid to such characteristics and further established among a public audience.

\section{Interviews}

Due to limited participants being selected for the interviews, they were withheld from the breadth of the analysis; however, they were included for the purposes of potential further research. Four participants were interviewed and around 10 general questions were asked, with follow up questions asked as needed. These participants were asked questions around the premise of the effect of high school on their physical and mental appearance and the outcomes align with the results of the survey. All interviews were recorded for the purposes of research.

When looking at the pressures placed on mental health, all participants responded with similar answers of how their main factor was under the belief of school or school related activities. Participant 1 was recorded stating that they felt major pressure due to school work, which was directly affecting their mental health. They felt, "due to society", that they were required to take certain classes, even though it did not seem to be what they truly wanted. Participant 3 noted that while school required functioning students to continuously attend on a daily basis, it is close to impossible for students to keep up with the strenuous classes that society often expects of them. During a period of time where students are continuing to learn what they are truly comfortable with and what they are not, they are often pushed into taking classes that could potentially ruin their GPA, which is a detrimental problem. Participant 3 further continues that due to one class that a school counselor all but told him/her to take, his/her GPA was ruined for the rest 
of high school, which was one of the key reasons that their mental health took such a toll in high school. They even stated that they were formally diagnosed with depression, something that became a large part of his identity.

Social pressure was also seen to exist in the area regarding weight class. While none of the participants were seen to be on extreme sides of the weight class scale, participant 2 specifically notes that they felt pressure to gain weight in order to fit into what society deemed as correct. They continued that due to family and societal pressure, they had to go through "a lot to gain around 20 pounds", which fit what was deemed to be the "correct weight." Participant 4 also seemed to find the same pressure exerted on them, which became an environment where how they appeared was a large part of why they felt insecure on a daily basis.

In the process of the interviews, a potentially suicidal participant was found, in which preventive measures were enacted to ensure the mental health of the participant.

This qualitative data collected was significant as while it clarified the hypothesis, it allowed for a more accurate description to test the correlation between BMI levels and self-reported depression levels. The interviews confirmed that a correlation does exist, seeing that in many situations, societal and school pressure forces many to feel like they must appear a certain way, becoming a large factor into depression or anxiety they may be feeling. This is a bidirectional relation, where the societal pressure enforced a standard for mental and physical health often decreased the other.

\section{Limitations}

After conducting the research, a few limitations were found that limited the scope and probability of results. One of the major limitations was the factors used to distinguish weight class levels and obesity. A study conducted by the Department of Endocrinology and Metabolism ${ }^{19}$ in Austria found that "BMI incorrectly classified normal athletes as fat," mischaracterizing the fat patterns and muscle mass. While other methods to distinguish weight patterns at a large scale exist, BMI mischaracterizes a large amount of the tested population. Due to the limited resources of the research, it was constricted to a survey-based study. However, should further studies allow, BMI should not be the preferred method to determine the correlation of obesity, instead preferring methods such as bioelectrical impedance or densitometry ${ }^{20}$.

Another large limitation of the data population was the limited demographics seen. With the data population, there was a limited amount of people fitting under the different demographics. This limitation created a lower range of results to be able to better understand a few specific correlations. If more participants were seen in different race or income categories, more diverse results could be found.

Due to the lack of participants for the interviews, they had to be excluded from the breadth of the study. This important correlation between societal perception and obesity levels between schools can serve to bring important insight. However, not enough participants agreed to be interviewed, failing to allow for there to be conclusive results to the overall paper. All in all, with a university affiliation and a resource docket, this study can be expanded to discard the aforementioned limitations and be used to advance the field of psychology and health.

\section{Conclusion}

Overall, this research study was inconclusive in relation to the question: to what extent does the correlational effect between self-reported depression and BMI levels play a factor at a suburban high school? The direct correlation was seen to be $4.2 \%$, with limiting factors stopping the breadth of the research. This study shows that there is no bidirectional correlation, however, proved further aspects that need to be further researched. With the small relation between particular races and household incomes, this unknown correlation may exist on a more individualized basis. Results were found, filling the much necessary gap in the field of research. 
Along with the stigma behind the topic, more can be done to explore the idea of societal pressure on rising depression and obesity levels. Further research has to be conducted in order to fully examine the link between what has been found and what can be inferred. Research specified towards certain minority or lower societal status groups can have potentially higher correlations between BMI and depression. The limited scope of the study brings into question: do certain factors have a higher effect on depression and obesity levels? Answering this question can lead to better ramifications on how to deal with the rising levels of depression in the status quo. More research can specify the larger effects of BMI in relation to SES groups and can help to see what groups are more in danger of higher depression levels.

Analyzing the effects of societal pressure and stigma between these factors has a research depth of potentially interesting findings. With a larger breadth of interviewees, more research ought to be conducted on the specific effects, bringing importance to the idea of how society and morals affect how people should look or feel. With participants feeling the effects of schools on their mental and physical health, a wider scope of people can see the correlation between school depression/weight. This important study can help shed light on larger issues, such as rising suicide rates. If this problem is tackled at high schools, adolescent depression and BMI may be solved at a local level, without long term effects.

\section{Acknowledgments}

I would like to primarily thank my mentor, Kelly Gorton, for her aid in procuring resources and mentorship throughout this tedious process. I would also like to express gratitude towards Justin McCrackin for his instruction with this research study. I would like to acknowledge Michael Horn in his teachings in the AP Capstone Program. Last of all I would like to thank my parents for their patience and help in the making of this research.

\section{References}

1. Schwartz, B. S., Glass, T. A., Pollak, J., Hirsch, A. G., Bailey-Davis, L., Moran, T. H., \& Bandeen-Roche, K. (2016, December). Depression, its comorbidities and treatment, and childhood body mass index trajectories. https://www.ncbi.nlm.nih.gov/pmc/articles/PMC5125866/.

2. Sodhi, V. (2007). Teenage Depression. Hinduism Today, 29(3), 62. Retrieved from http://search.ebscohost.com/login.aspx?direct $=$ true $\& \mathrm{db}=\mathrm{asn} \& \mathrm{AN}=25752016 \&$ site $=$ ehost-live

3. Goodwin, R. (2017, October 30). Depression is on the rise in the US, especially among young teens. Retrieved from https://www.sciencedaily.com/releases/2017/10/171030134631.htm.

4. Rice-Oxley, M. (2012, March 14). The truth about depression: six people speak out. Retrieved from https://www.theguardian.com/society/2012/mar/14/truth-about-depression.

5. Lewinsohn, Peter M., Greogory N. Clarke, John R. Seeley, Paul Rohde, Major Depression in Community Adolescents: Age at Onset, Episode Duration, and Time to Recurrence, Journal of the American Academy of Child \& Adolescent Psychiatry, Volume 33, Issue 6, 1994, Pages 809-818, ISSN 0890-8567, https://doi.org/10.1097/00004583-199407000-00006.

6. Assaad, S., Anouti, S., Naja, F., Nasreddine, L., Hwalla, N., Sibai, A. M., Child: Care, Health \& Development, 03051862, Jan2018, Vol. 44, Issue 1 
7. Borchard, T. (2018). Why Are So Many Teens Depressed?. Psych Central. Retrieved on December 11, 2019, from https://psychcentral.com/blog/why-are-so-many-teens-depressed/

8. Glied S, Pine DS. Consequences and Correlates of Adolescent Depression. Arch Pediatr Adolesc Med. 2002;156(10):1009-1014. doi:https://doi.org/10.1001/archpedi.156.10.1009

9. “Overweight \& Obesity Statistics." National Institute of Diabetes and Digestive and Kidney Diseases, U.S. Department of Health and Human Services, 1 Aug. 2017, https://www.niddk.nih.gov/health-information/healthstatistics/overweight-obesity.

10. Chiang, I.-C. A., Jhangiani, R. S., \& Price, P. C. (2015, October 13). Correlational Research. Retrieved from https://opentextbs.ca/researchmethods/chapter/correlational-research/

11. Strickland, J. (2017, December). Building a better survey experience. Retrieved from https://www.apa.org/science/about/psa/2017/12/survey-experience.

12. Grohol, J. M. (n.d.). Depression Test - Do You Have Depression? Retrieved from https://psychcentral.com/quizzes/depression-quiz/.

13. "Adult BMI Calculator." Centers for Disease Control and Prevention, Centers for Disease Control and Prevention, 19 Aug. 2019, www.cdc.gov/healthyweight/assessing/bmi/adult bmi/english bmi calculator/bmi calculator.html.

14. U.S. Census Bureau (2017). "U.S. Median Household Income Up in 2018 From 2017." The United States Census Bureau, US Department of Commerce, 29 Oct. 2019, www.census.gov/library/stories/2019/09/us-medianhousehold-income-up-in-2018-from-2017.html.

15. U.S. Census Bureau (2018). American Community Survey 1-year estimates. Retrieved from Census Reporter

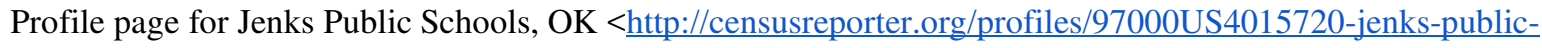
$\underline{\text { schools-ok/> }}$

16. "Office of Minority Health." Obesity and Hispanic Americans - The Office of Minority Health, Center of Disease and Control, 2016, minorityhealth.hhs.gov/omh/browse.aspx?lvl=4\&lvlid=70.

17. Asiseh F, Yao J. Family income and body mass index - what have we learned from China. Health Econ Rev. 2016;6(1):52. doi:10.1186/s13561-016-0129-z

18. Morgenstern, M., Sargent, J. D., \& Hanewinkel, R. (2009). Relation between socioeconomic status and body mass index: evidence of an indirect path via television use. Archives of pediatrics \& adolescent medicine, 163(8), 731-738. doi:10.1001/archpediatrics.2009.78

19. Kruschitz, R., Wallner-Liebmann, S. J., Hamlin, M. J., Moser, M., Ludvik, B., Schnedl, W. J., \& Tafeit, E. (2013). Detecting Body Fat-A Weighty Problem BMI versus Subcutaneous Fat Patterns in Athletes and NonAthletes. PLoS ONE, 8(8), 1-9. https://doi.org/10.1371/journal.pone.0072002 
20. "Measuring Obesity." Obesity Prevention Source, Harvard School of Public Health, 12 Apr. 2016,www.hsph.harvard.edu/obesity-prevention-source/obesity-definition/how-to-measure-body-fatness/.

21. Berry, T. R., Spence, J. C., Blanchard, C., Cutumisu, N., Edwards, J., \& Nykiforuk, C. (2010, February 16). Changes in BMI over 6 years: the role of demographic and neighborhood characteristics. Retrieved from https://www.nature.com/articles/ijo201036

22. Fokeena, W. B., \& Jeewon, R. (2012). Is there an association between socioeconomic status and body mass index among adolescents in Mauritius?. TheScientificWorldJournal, 2012, 750659. doi:10.1100/2012/750659.

23. Byrne, M., O’Brien-Simpson, N., Mitchell, S., Allen, N., Byrne, M. L., O’Brien-Simpson, N. M.... Allen, N. B. (2015). Adolescent-Onset Depression: Are Obesity and Inflammation Developmental Mechanisms or Outcomes? Child Psychiatry \& Human Development, 46(6), 839-850. https://doi.org/10.1007/s10578-014-0524-9

24. Respress, B. N., Morris, D. L., Gary, F. A., Lewin, L. C., \& Francis, S. A. (2013). Social Determinants of Adolescent Depression: An Examination of Racial Differences. Issues in Mental Health Nursing, 34(7), 539-549. https://doi.org/10.3109/01612840.2012.758206

25. Devenish, B., Hooley, M., \& Mellor, D. (2017). The Pathways Between Socioeconomic Status and Adolescent Outcomes: A Systematic Review. American Journal of Community Psychology, 59(1/2), 219-238. https://doi.org/10.1002/ajcp.12115

26. Tashakori, A., Riahi, F., \& Mohammadpour, A. (2016). The Relationship between Body Mass Index and Depression among High School Girls in Ahvaz. Advances in medicine, 2016, 3645493.

https://doi.org/10.1155/2016/3645493 